\title{
Can portion size and energy density estimates be influenced by perceived "healthiness" of foods?
}

\author{
G. P. Faulkner, L. K. Pourshahidi, J. M. W. Wallace, M. A. Kerr, T. A. McCrorie \\ and M. B. E. Livingstone \\ Northern Ireland Centre for Food and Health (NICHE), University of Ulster, Cromore Road, Coleraine, BT52 1SA, UK
}

Foods approved for claims such as 'reduced fat' and 'reduced energy' have been advocated as a strategy to counteract excessive energy intake. However, such foods may be perceived by consumers as "healthier" than their standard counterparts, which may lead to inappropriate portion size selection ${ }^{(1,2)}$. The aim of the current study was to compare portion size estimates, perceived energy content, and anticipated consumption guilt of "healthier" $v s$. standard foods.

Three pairs of isoenergy dense $(\mathrm{kJ} / 100 \mathrm{~g})$ foods, i.e. a perceived "healthier" cereal, drink and coleslaw vs. a standard cereal, drink and coleslaw were displayed in two separate rooms. For each food, subjects were asked to estimate (by serving out) an appropriate portion for themselves and estimate the energy content of that portion. Room order was randomised and questions were asked in systematic rotation within each room. In addition, subjects were asked to rate how guilty they would feel after consuming their estimated portions of each food on a scale of 1 (not at all guilty) to 5 (very guilty). Estimated portions were weighed and recorded after subjects left the room.

In total 186 adults completed the protocol: $52 \%$ female, mean (SE) age $32(0.96)$ years, and mean (SE) BMI 26.4 (0.3) kg/m². Subjects estimated larger portions of the "healthier" coleslaw than the standard version $(P<0.001)$, and perceived the "healthier" foods to be lower in energy density than the standard foods $(P<0.001)$ even though they were isoenergy dense. Higher anticipated consumption guilt was associated with the standard foods (means: 2.3 vs. $1.3 ; P<0.001$ ). Similar results were observed between genders and BMI categories.

\begin{tabular}{|c|c|c|c|c|c|c|c|}
\hline \multirow[b]{2}{*}{ Portion size (g) } & \multicolumn{3}{|c|}{ "Healthier" } & \multicolumn{3}{|c|}{ Standard } & \multirow[b]{2}{*}{$P$ value* } \\
\hline & Median & 25 th & 75 th & Median & 25 th & 75 th & \\
\hline All foods & 133 & 109 & 167 & 129 & 103 & 164 & 0.307 \\
\hline Drink & 255 & 203 & 344 & 260 & 200 & 356 & 0.556 \\
\hline Cereal & 49 & 38 & 65 & 49 & 34 & 64 & 0.198 \\
\hline Coleslaw & 77 & 55 & 106 & 73 & 53 & 95 & $<0.001$ \\
\hline \multicolumn{8}{|c|}{ Energy Density $(\mathrm{kJ} / \mathbf{1 0 0} \mathrm{g})$} \\
\hline All foods & 560 & 315 & 876 & 831 & 553 & 1284 & $<0.001$ \\
\hline Drink & 143 & 83 & 225 & 193 & 106 & 327 & $<0.001$ \\
\hline Cereal & 985 & 514 & 1674 & 1416 & 825 & 2152 & $<0.001$ \\
\hline Coleslaw & 477 & 225 & 837 & 828 & 465 & 1231 & $<0.001$ \\
\hline
\end{tabular}

*Significant difference between "healthier" and standard groups $(P<0.05$, Wilcoxon signed rank tests).

Despite perceptions of standard foods being higher in energy, and causing a greater anticipated consumption guilt compared to the perceived "healthier" foods, consumers failed to compensate for this by selecting a smaller portion size. In terms of food choice, the complex interaction between knowledge and behaviour change is highlighted by this study. Future studies should include a wider range of foods to confirm these findings.

This material is based upon works supported by safefood, the Food Safety Promotion Board, under Grant No. 07-2010. Ethical approval was obtained from the University of Ulster Research Ethics Committee and the study was conducted according to the guidelines laid down in the Declaration of Helsinki.

1. Wansink B \& Chandon P (2006) J Marketing Res, 43, 605-617.

2. Kelly M, Wallace J, Robson P et al. (2008) Obesity, 16 (Suppl. 1). 